\title{
Hugo Francisco Bauzá
}

\section{Ln célébration des festivités indigènes (le carnaval et la Pacha Mama) Jans le Nord-Ouest argentin comme murs défensifs foce à ln mondialisation}

The Celebration of Indigenous Festivities (THE CARNival AND the PaCha Mama) IN NorthWESTERn ARGentina AS Defensive WALLS AGAINST GLOBALIZATION

Abstract: Although philosophers agree that the ego is formed only in relation to others, that social groups, peoples and nations are the result of prolonged and inseparable interbreeding which makes it possible to speak of multiple identities - either multiculturalism or interculturalism - the natives of northwestern Argentina (NOA) strive to preserve their cultural identity marks. Keywords: Argentina; Globalization; Cultural Identity; Celebration; Carnival; Pacha Mama; Rites; Festivity.

\section{Hugo Francisco BaUzá}

Académie Nationale de Sciences de Buenos Aires hfbauza@yahoo.com.ar

DOI: 10.24193/cechinox.2021.40.12
T Quel serait le sens aujourd'hui pour - nous, individus soit disant rationnels, de faire attention à deux anciennes festivités des Andes tel que le rituel du "Carnaval » et celui du culte à "La Pacha Mama », célébrations soit disant irrationnelles?

Face au néocapitalisme sauvage qui secoue l'Occident actuel, si nous considérons ces rites et cultes ancestraux, nous devrions peut-être inverser la signification des termes rationnel / irrationnel et nous demander dans quelle mesure la culture des populations primitives du NordOuest argentin (NOA) peut nous orienter pour donner un sens au rapport identité - développement et arriver à habiter le monde non pas de manière tellement artificielle, tel la modernité occidentale, mais d'une manière plus authentique.

La survivance de la culture de ces populations, tout au long des siècles, fait preuve de la force et de la résistance de ses valeurs traditionnelles, malgré l'effort constant des envahisseurs pour coloniser culturellement l'Amérique indigène, comme l'ont remarqué Adolfo Colombres ${ }^{1}$ 
chez les anciens habitants du NOA et Serge Gruzinski ${ }^{2}$ chez les habitants du Mexique.

Ces festivités, comme elles sont célébrées dans des lieux éloignés des centres urbains, sont comme des " marges » et étant donné cette distance il paraît qu'elles mènent une vie plus naturelle et conçoivent la réalité de manière plus harmonieuse. Ce sont des espaces de transformation comme a remarqué Victor Turner en considérant des notions de structure et contre-structure des phénomènes rituels ${ }^{3}$. Dans ces célébrations, tout en fixant une identité culturelle, on bouleverse l'ordre hiérarchique du monde imposé par l'exercice du pouvoir dominant, et on arrive à proposer une existence parallèle à travers laquelle on résiste face à toute déformation des traits identitaires.

Dans ce sens, on conçoit ces pratiques comme une sorte de "décompression " de conflits sociaux. On se demande en même temps si ces rites peuvent réveiller dans notre conscience une lueur du temps primordial où l'homme, avec une vision non-rationnelle du cosmique, comprenait la vie comme sacrée.

Ces rituels peuvent-ils, pendant la fête, à la fois, renforcer la dimension identitaire de ceux qui la célèbrent et donner la lueur d'un autre mode de vie ?

De nos jours, il est nécessaire de faire attention au savoir des sociétés traditionnelles qui refusent d'accepter la temporalité linéale de l'histoire. Dans ce sens, les rituels du NOA nous apprennent que la vie a d'autres niveaux de perception, niveaux qui vont au-delà des mathématiques et de la logique classique. Autrement dit, ils nous suggèrent de ne pas nous tenir exclusivement à une pensée binaire - rationnel vs. irrationnel - qui oublie quentre blanc et noir il y a une quantité infinie de gris ${ }^{4}$; de plus, ils nous apprennent à nous tenir à la permutabilité de ces concepts, et à être attentif à l'existence d'autres modes d'appréhension de la réalité.

Dans une logique classique l'état d'une monnaye est pile ou face, peu importe l'observation que nous faisons de celle-ci. La physique quantique, en revanche, admet que l'état d'une pièce, ou de n'importe quel système quantique, est une superposition ou mélange de différents états tant qu'on n'intervienne pas avec notre point de vue ; de ce fait, « le système se trouve dans une sorte de limbe de probabilités où les deux états cohabitent $»^{5}$. Dans cette dimension, certaines traditions mythiques évoluent indépendamment du fait historique vérifiable. C'est seulement en faisant l'observation qu'apparaît un état " pur ", bien défini, en relation à une certaine probabilité. Dans ce sens, c'est l'homme qui en tire une conclusion basée sur des évidences disponibles.

De la même manière qu'on fait une expérience en physique, ce serait hasardeux de décider quelles épreuves seront efficaces Et de cette manière on tranche entre ce qui pourra être accepté comme réalité prétendument objective et ce qui pourra être accepté comme mythe, c'est-à-dire une réalité qui manque d'explication rationnelle puisque sa nature obéit à un autre registre. Chaque culture est un monde, protégé dans ses rites et ses croyances, et c'est pour cela que nous ne pouvons pas nous limiter à une seule interprétation, on a besoin de différentes lectures.

L'étude des fêtes nous fait découvrir d'autres expressions de vie. En ce qui concerne le sens de la fête, en tant que 
divertissement, il vaut mieux remarquer le sens du mot, dérivée du verbe latin diuertere, "diverger, aller sur de différents chemins ", qui nous suggère un raccourci pour échapper à une mondialisation aliénante. Les sociétés traditionnelles, ayant un fort sens du social et de la communauté, peuvent nous proposer de nouvelles manières pour penser le monde.

Les rites inaugurent un espace-temps idéal un cadre qui nous éloigne du réel et nous fait oublier la notion de finitude. Dans la fête se trouve la mémoire culturelle d'une société6. Dans le cas de la fête du NOA, l'imaginaire social est formé d'un ensemble de croyances, de rituels et d'autres pratiques symboliques qui, modèlent la psyché et l'identité, et, en outre, prétend l'éclairer par rapport au mystères de l'existence.

Dans ces rituels, spécialement dans celui de la Pachamama, la valorisation du féminin est un aspect clé. De plus, ces fêtes favorisent le tourisme promu par des politiques publiques et participent à la mise en valeur du patrimoine symbolique. En ce sens, la spécialiste María Elena Babino a mis en relief le rôle des universités locales dans la relation entre la culture et le tourisme en Argentine ${ }^{7}$, aspect étudié dans le dossier " Débats autour de l'Anthropologie de l'État et les Politiques Publiques dans des contextes latino-américains contemporains ${ }^{8}$.

Aujourd'hui, ce patrimoine identitaire est soutenu par divers musées et archives qui le préservent, et le rendent visible. On signale le travail récent sur les ermitages de Tilcara de Graciela C. Sarti ${ }^{9}$.

Les festivités de cette culture sont la garantie de l'identité et de la cohérence, face aux autres cultures ; ces festivités uniques sont aussi une fable collective qui illustrent une conception particulière de la vie.

Comment expliquer la vie d'autrui ? Comment se voir et raconter les habitudes et les coutumes des anciens habitants du Nord-Ouest argentin à partir des catégories mentales de notre imaginaire?

Dans la fête on peut entrer en contact avec une altérité transcendantale. Dans cette culture on assiste à l'interaction entre l'environnant naturel et l'environnant social, ce que vient d'expliquer, avec lucidité, Philippe Taupin.

Nous nous demandons s'il est possible, à partir de notre imaginaire mental, de pénétrer dans l'imaginaire symbolique de ces peuples, de comprendre la manière dont ils sentent le sacré en tant qu'expérience sensible de la transcendance (le caractère numineux dans les termes de Rudolf Otto).

II. La sigle NOA désigne une région du nord-ouest argentin comprenant les provinces de Jujuy, Salta, Tucumán, La Rioja et - pour de raisons historiques et non pas géographiques - une partie de Santiago del Estero. Les anthropologues $\mathrm{y}$ ont trouvé quelques vestiges de populations chasseuses-cueilleuses qui datent $\mathrm{du}$ 8500 avant Jésus-Christ.

On imagine qu'à partir du 3000 avant Jésus-Christ on y aurait commencé un processus de sédentarisation grâce à l'agriculture. Déjà dans notre ère - entre 1200 et 1400 - on peut constater l'influence de la culture du Tiahuanaku, une ville archéologique dans le département de La Paz, en Bolivie. Il s'agit d'une culture pré-inca fondée sur l'agriculture et l'élevage. Celle-ci est originaire du territoire de l'actuelle Bolivie, autour du lac Titicaca, mais elle 
s'est étendue jusqu'au nord de l'Argentine, du Chili et au sud du Pérou. Parmi les précieux témoignages on peut trouver la Port du Soleil, une sculpture monolithique ayant une dimension rituelle et astronomique. Cette culture a été dominée, vers le XIII ${ }^{\mathrm{e}}$ siècle, par les Inca qui venaient de l'actuel territoire du Pérou (l'archéologie a remarqué, entre autres, ses empreintes dans la construction du kapar ñan, " le grand chemin » ou " le chemin des Inca », aujourd'hui important non seulement pour les anthropologues et historiens, mais aussi pour l'UNESCO ${ }^{10}$ ).

Les Inca ont établi leur centre administratif à Cusco, une ville située sur l'altiplano andin ; le gouvernement des inca a été abattu en 1553 par les espagnols conduit par Francisco Pizarro. Celui-ci, profitant du désaccord des gouvernants - les frères Huáscar et Atahualpa, les fils de l'Inca - a tendu une embuscade et puis a mis en prison et a tué Atahualpa. L'apparition des Inca a été dévastatrice pour cette culture, ainsi que pour plusieurs anciennes populations. On a détruit leurs pucaraes "forteresses » et l'ancien cimetière des diaguitas - éloignés de Cusco - afin d'anéantir leur passé puisque ces populations rendaient hommage à leurs ancêtres. De plus, les inca ont prétendu remplacer leurs croyances traditionnelles par une religion d'État au centre de laquelle était l'Inca, l'homologue du Soleil.

Ils ont soumis les anciennes populations de différentes manières, notamment en les obligeant à payer des contributions pour les travaux agricoles ; cependant, malgré leur pouvoir, ils ont été disloqués par les Quechua et les Aimara. Vers la moitié du $\mathrm{XVI}^{\mathrm{e}}$ siècle, les habitants du NOA ont connu l'arrivée des espagnols, qui en 1553 ont débarqué au Chili en apportant avec eux des maladies du Vieux Continent (la rougeole, la variole et diverses souches de grippe).

Ceux-ci ont contribué à la mise en place de villes capitales qui existent encore de nos jours. On assiste peu à peu à la différentiation entre les envahisseurs hispaniques d'un côté et les aborigènes de l'autre côté ; ceux derniers ont lutté pour la conservation de leurs habitudes leur langue, leur religion et leurs rites - et surtout, de leur liberté. Il y a eu diverses confrontations entre les aborigènes et les Espagnols, dont la plus connue et celle du dernier Inca, le cacique Tupac Amaru, soumis à un sparagmós - sacrifice rituel de la part des espagnols en 1572.

La création du Vice-royauté du Pérou, siégé à Lima, a été un événement politique de grande importance. Celui-ci occupait un énorme espace du territoire ; de l'Argentine actuelle il comprenait deux régions $\mathrm{du}$ NOA : l'administration de Salta del Tucumán et celle de Córdoba del Tucumán.

La cohabitation des espagnols et les anciens habitants a entraîné la naissance des populations métisses. Pourtant, ce mélange n'a pas supposé la disparition des populations originaires qui ont pu conserver leurs fêtes et leurs traditions. De ces groupes on peut trouver notamment les Coyas $^{11}$ - descendants des anciens Atacameños -, les Chichas, les Diaguitas et, entre autres, les Omaguacos. En dépit de l'arrivée des missionnaires - franciscains, jésuites et dominicains - qui ont répandu leur dogme en langue quechua, cette circonstance n'a pas impliqué l'extinction des rites originaires. Ceux-ci ont persisté à côté du christianisme prêché par les évangélisateurs, d'autant plus que, dans certaines régions, il y a eu un mélange de cultes. 
Dans cette région géographique se trouve la Quebrada de Humahuaca, dans la province de Jujuy, une profonde fissure d'origine tectonique et fluvial encaissé par les pics des montagnes de la précordillère des Andes, espace retiré à cause de son accès difficile, ce qui lui a permis de cultiver des anciennes traditions. On peut remarquer que dans cette région montagneuse du NOA on trouve des sites géographiques vraiment originaux; notamment la « $\mathrm{La}$ pente de l'évêque " à Salta, "La montagne aux sept couleurs ", à Purmamarca et à Jujuy le Parc National des cardones, une région désertique de plus de $60.000 \mathrm{hec}-$ tares qui fait partie du Patrimoine Mondial de l'Humanité de l'UNESCO.

Malgré l'évidente influence espagnole de presque 500 ans, la région du NOA préserve encore de très vivants rituels ancestraux, comme celui de la Pacha Mama "Mère Terre » et celui de la festivité du Carnaval. Ces festivités, resémantisées par les tableaux du peintre A. Gramajo Gutiérrez, d'où émergent leurs projections imaginaires, s'imposent comme des murs défensifs de leur identité face à une uniformité compulsive et aux tensions centripètes de la mondialisation dénoncées par le théoricien de l'art, le paraguayen Ticio Escobar ${ }^{12}$.

\\. $\begin{aligned} & \text { Quant au carnaval, c'est une } \\ & \text { cébration publique qui a lieu }\end{aligned}$ immédiatement après le carême chrétien. C'est un rituel lié aux festivités commémoratives de nature cosmogonique : c'est la fête vécue comme libération des passions semblable à bien des égards au dionysisme cultivé par les Grecs ou les Saturnales des Romains. Il dure généralement trois jours, ce que l'État argentin reconnaît officiellement comme des vacances, bien que parfois cette période puisse être prolongée si l'on tient compte des préparatifs. Bien qu'ils soient célébrés dans différentes villes andines, les carnavals de la Quebrada de Humahuaca, Tilcara et Maimará, dans la province de Jujuy, jouissent d'une plus grande renommée, où deux mondes parallèles vivent ensemble : celui de la réalité quotidienne et celui de de la fête. De même les fêtes "Las carpas ", dans la vallée de Lerma, dans la province de Salta, sont aussi célèbres ; un espace symbolique fortifié par des habitudes et des coutumes ancestrales. Le carnaval déterritorialise le discours politique dominant en proposant une sorte d'immunologie quant au social et au politique.

Le festival consiste en le déterrement - le «Grand Carnaval »- puis l'enterrement - «Petit Carnaval » - du Pujllay, « le diable du Carnaval ", ressenti comme le soleil, représenté par une poupée ; ancienne fête thaumaturgique - une immanence magique - liée à des rites de fertilité primitifs basés sur les équinoxes et les solstices qui déterminent les saisons de l'année, c'est-à-dire une fête soumise à des rythmes primordiaux. Ce festival a été célébré dans le NOA avant l'arrivée des Espagnols et continue d'être célébré même avec certains éléments culturels des envahisseurs, que nous détaillerons plus tard. Le festival se termine le «Dimanche de la tentation " lorsque le diable est enterré avec des feuilles de coca et d'autres éléments rituels. Pour l'imaginaire populaire, le diable du carnaval est celui qui féconde la Pacha Mama " Mère Terre ", en favorisant les cultures. Lors de sa célébration, les désirs refoulés sont libérés, notamment ceux liés au sexe. On y aplanit les différences sociales : un vent de licence, d'anarchie 
transitoire, de débordement fantastique. « Notre seule liberté était les six jours du carnaval, rien de plus » vient de déclarer Ivana Tintilay ${ }^{13}$, chercheuse le jour et travailleuse du sexe la nuit. Ses recherches ouvrent la voie à l'étude des problèmes de genre et de leurs multiples dérivations dans les processus politiques de la post-dictature en relation avec la culture du NOA. Après le festival, neuf mois plus tard naît un enfant et on le considère comme le « fils de la fête ": "Carnaval que vous vouliez, prenez le carnaval et à neuf mois un bébé (créature) à porter ", dit un couplet. Il s'agit du retour à un dionysisme primitif, corroboré par une sorte d'orgie multiforme qui conduit les célébrants à se fondre dans des expériences de groupe. Une intoxication de transgression y a lieu, condition d'émancipation et du dépassement de toute limite de l'existence, selon l'opinion de J.-J. Wunenburger ${ }^{14}$; un débordement de l'irrationnel, symbole d'un comportement libertaire collectif. Raúl García Rodríguez, s'appuyant sur Michail Bakhtine ${ }^{15}$, défend la « carnivalisation du monde comme pratique de résistance et de transformation sociale face à l'hégémonie d'un dialogue établi ou d'un discours dominant et institutionnalisé ${ }^{16}$; en ce sens le carnaval est présenté comme un rituel cathartique ou thérapeutique qui tente de restaurer les déséquilibres sociaux. Lorsque nous parlons de dionysisme, nous ne nous référons pas spécifiquement à la religion de la divinité mythique grecque, mais à une figuration symbolique, multiforme et subtile, à travers laquelle se met en place une fusion cosmique. Pendant le carnaval, tout le monde peut incarner Pujllay puisque s'y produit la libération des liens formels et, en outre, la consommation de boissons alcoolisées et d'autres substances, certaines hallucinogènes, provoquent une certaine ferveur. Jeux de rôles, jeux de substitution dans lesquels le mythe et le rite véhiculent la sacralité originale. C'est le moment où éclatent la débauche et le rire guérisseur, un rire libérateur. Aussi, en raison de sa nature rédemptrice, le festival cherche à nous éloigner de nos obsessions et de nos peurs. Bien qu'il s'agisse de festivités païennes, elles comprennent également des éléments étrangers apportés par la conquête hispanique, par exemple, dans la ville de Casabindo (dans la province de Jujuy), le 15 août, la fête patronale de l'Assomption de la Vierge, «la corrida du bandeau », dans laquelle, contrairement à ce qui se passe en Espagne, l'animal n'est pas blessé. Le festival a en centre le torero qui doit saisir les pièces de monnaie que l'animal porte dans un bandeau entre ses cornes. Cette cérémonie spécifique et identitaire de la ville de Casabindo fait venir chaque année plus d'un millier de personnes sur ce territoire perdu de l'Altiplano, ce qui dynamise le tourisme et l'économie de la région; de plus, cette fête populaire est enrichie par la musique, les danses, les boissons et les plats régionaux. Cette tradition explique l'effort de la population de défendre fermement ses traditions contre la migration de ses habitants à la recherche du travail.

】 . la "Terre Mère " est sans aucun doute la croyance mythologique la plus répandue dans la région NOA. La Pacha Mama est la divinité féminine de la terre qui nourrit et protège. La notion de nature divinisée entretient un certain rapport avec l'idée du saint d'Assise ou avec celle des mystiques qui proclament la relation 
immanente de la divinité et du monde, comme si la nature était animée par une force incompréhensible. La Pacha Mama est, fondamentalement, une divinité protectrice dont le culte s'étend sur toute la côte andine. Le rituel en son honneur est une fête tellurique qui se déroulée - et se pratique encore aujourd shui - au début des semailles et de la récolte ; le jour clé est le $1^{\text {er }}$ août lorsque les autochtones lui offrent de la nourriture, des boissons et des feuilles de coca déposées dans un pot d'argile qu'ils enterrent en son hommage ${ }^{17}$. Ces offrandes constituent la challa ou le paiement, une sorte de tribut en faveur de la divinité, semblable au troc du paganisme. Si ces offrandes ne sont pas faites, la Pacha Mama est offensée et peut causer des dommages. Elle est toujours présente et les habitants entretiennent un dialogue permanent avec elle. La terre est vue dans comme dans la pensée pré-socratique qui la liait au cosmos et à l'homme ; ainsi, en lui faisant sentir qu'il fait partie du cosmos, la fête rapproche l'homme du mystère de la vie elle-même. Cette vision du monde contraste avec celle que les Espagnols ont apportée en Amérique à partir de la Renaissance où l'homme, par sa raison, cherche à se séparer de la phýsis en devenant un sujet autonome, c'est-à-dire en dehors de la vision cosmique des primitifs, initiant ainsi un processus progressif de sécularisation où l'homme est en possession de soi-même.

De cette manière, l'Europe néglige l'aspect écologique de l'univers, ne s'occupant que de son aspect matériel. Cela ne va pas de même pour les Coyas pour qui la mort n'implique pas la finitude absolue, mais l'entrée dans un autre état d'existence. Face au démembrement historique de la culture moderne, les habitants primitifs du NOA, avec leurs rites et traditions ancestraux, conçoivent la vie de telle manière que l'âme individuelle se sentait participante au mystère du cosmique. Dans le monde moderne, sous l'influence de la pensée cartésienne, l'Occident européanisé se distancie du caractère vivant de la nature, ne la comprenant que comme chose inanimée, mesurable, et comme objet d'analyse rationnelle. Cette circonstance s'est aggravée lorsque la révolution industrielle, dans son désir démesuré de domination, lança sa conquête et son exploitation, fixant son regard non pas sur le qualitatif, mais sur le quantitatif, comme l'a bien dit René Guénon ${ }^{18}$.

Le catholicisme apporté par les conquérants a tenté de s'imposer de façon impériale, détruisant les mythes et les religions ancestrales, et séparant l'homme de sa vision cosmique primitive. Cependant, face à cette imposition, une réaction métaphysique de l'ancien panthéisme américain a eu lieu; celle-ci a vu - et voit - dans la Terre Mère une manifestation du sacré. Il s'agit de ce que l'anthropologue Günther Rodolfo Kusch, en parlant de la pensée indigène et populaire américaine, a nommé le « savoir être », c'est-à-dire ce qui existait avant d'assumer consciemment la tâche de prendre en charge sa propre existence ${ }^{19}$. Pour ce savant, la découverte du nouveau monde soulève la rencontre de deux expériences humaines : celle de lêtre - en tant qu'existence- et celle de lêtre - en tant qu'état -, la première étant celle de l'Europe du $X V I^{e}$ siècle et celle de létat, celle des cultures amérindiennes avant l'arrivée des Espagnols. Pour la pensée indigène, la vie a des aspects qui frisent la magie ; pour l'Européen, au contraire, tout doit 
être expliqué par une raison critique. De cette manière, Kusch distingue une réalité de forme (l'apparence de l'être européen) et une réalité de fond (qui concerne le tellurique et l'indien), ce qui lui permet de renverser le binôme traditionnel civilisation / barbarie, considérant civilisation la culture primitive des anciens habitants de l'Amérique, et barbarie la vision des européens qui se forçaient à anéantir des cultes, des rites et des cérémonies ancestrales des peuples conquis.

L'homme andin, protégé par la Terre Mère, célèbre également d'autres phénomènes de la nature, comme le solstice d'hiver qui, dans l'hémisphère sud, est célébré tous les 24 juin, jour du passage de l'obscurité à la lumière. Pour cette fête, ils allument des feux de joie où ils incinèrent des objets désaffectés puis marchent rituellement sur leurs braises. Un moment singulier où la vie, surmontant la contingence quotidienne, acquiert un sens transcendant dans lequel les barrières qui séparent ce monde de l'autre sont brisées. Ainsi s'installe un temps sacré qui contraste avec le profane, selon la lecture dichotomique suggérée par Mircea Eliade. Dans le festival émerge l'être ancestral et profond de l'homme qui consolide son identité, fortifiée par la survie de ses langues vernaculaires. Peutêtre que cela, étant lié à leurs habitudes et à leurs coutumes traditionnelles, peut, face à la mondialisation qui nous accable, nous venir en aide à une époque pleine d'incertitudes.

\section{NoTES}

1. La colonización cultural de la América indígena, Buenos Aires, Ed. del Sol, Serie Antropológica, 2004.

2. La colonización de lo imaginario, trad. J. Ferreiro, Mexique, Fondo de Cultura Económica, 1991.

3. Le phénomène rituel, structure et contre-structure, Paris, PUF, 1990.

4. Face à la lecture binaire des genres grammaticaux de la langue espagnole il y a en aujourd'hui quelquesunes qui proposent d'inclure les pronoms personnel s elle - et son pluriel elles - au Dictionnaire de la RAE l'Académie signale que sa possible acceptation devrait répondre à ceux qui peuvent ne pas se sentir définis par les deux genres traditionnels.

5. Je remercie l'Académicien ingénieur Luis A. de Vedia pour cette référence.

6. Marcel Mauss, Les fonctions sociales du sacré, Paris, Minuit, 1968.

7. "Cultura y turismo en la Región del Noroeste Argentino. Circuito turístico de ferias y fiestas populares desde la perspectiva de las universidades locales”, en Turismo Cultural: anàlisi, diagnóstico y perspectivas de futuro, Actas de Congreso, B. Gomila Juaneda (ed.), vol. 2, tomo 2, pp. 517-531.

8. En RUNA, archivo para las ciencias del hombre, Buenos Aires, vol. 41, 2 (2020).

9. "Aportes para el estudio de las ermitas de Tilcara : del rito a la imagen", en El imaginario de las formas rituales (Hugo F. Bauzá, comp.), Buenos Aires, Facultad de Filosofía y Letras, 2011, pp. 23-38.

10. Dès 2014 Patrimoine Mondial de l'Humanité par l'UNESCO; dans l'actualité il existe un projet plurinational pour le revaloriser.

11. Le terme coya - ou ces variantes graphiques colla ou kolla - fait référence à l'ensemble syncrétique de peuples indigènes du NOA.

12. El mito del arte y el mito del pueblo. Cuestiones sobre el arte popular, Buenos Aires, Edit. Ariel, 1986.

13. I. Tintilay vient d'obtenir la bourse de recherche "Alberto Ghirardo", crée en hommage à cet homme politique et écrivain socialiste, avec son travail "Reconstruyendo fragmentos de la vida travesti en la post dictadura argentina”, cf. Buenos Aires, INFOBAE, 22.III.2020 
14. Cf. "Las figuras de Dioniso", en El imaginario en el mito clásico (Hugo F. Bauzá, compilateur), Buenos Aires, Academia Nacional de Ciencias de Buenos Aires, 2004, pp. 13-14.

15. La cultura popular en la edad Media y en el Renacimiento. El contexto de F. Rabelais, Madrid, Alianza, 2005.

16. "La carnavalización del mundo como crítica: risa, acción política y subjetividad en la vida social y en el hablar", en Athenea Digital, 13 (2) julio 2013, p. 121.

17. Le do ut des du paganisme ; cf. M. Mauss, Essai sur le don. Forme et raison de l'échage dans les sociétés archaïques, Paris, 1925.

18. Cf. Le Règne de la Quantité et les Signes des Temps, Paris, Gallimard, 1945.

19. Cf. América Profunda (Buenos Aires, Biblos, 1962) y Geocultura del hombre americano, Buenos Aires, García Cambeiro, 1976. 